The validity of the assumption of a crystalline field of almost perfect cubic symmetry can be tested with considerable accuracy by an appeal to magnetic data, since any deviation from cubic symmetry causes anisotropy in the paramagnetic susceptibility. At room temperatures, for the rare earths (anisotropy/ mean susceptibility) must be of the same order as (splitting caused by non-cubic terms in a triply degenerate cubic level/overall splitting due to cubic field). Prof. K. S. Krishnan has kindly informed us that $\mathrm{Pr}_{2}\left(\mathrm{SO}_{4}\right)_{3} \cdot 8 \mathrm{H}_{2} \mathrm{O}$ at room temperatures has an anisotropy roughly 10 per cent of the mean susceptibility ${ }^{2}$. Hence, separations of at least $50 \mathrm{~cm}^{-1}$ must be assumed between the various components of either of the two triply degenerate cubic levels of $\mathrm{Pr}+++$ in order to account for the magnetic measurements. All nine levels of $J=4$ should show up in absorption spectra, and the failure of Spedding to locate more than four may simply mean that at room temperatures these are the only ones with any appreciable population. Again, the explanation may very well lie in peculiar intensity rules, at present not understood.

We have made further calculations on the mean susceptibility and anisotropy of $\operatorname{Pr}_{2}\left(\mathrm{SO}_{4}\right)_{3} \cdot 8 \mathrm{H}_{2} \mathrm{O}$, and the corresponding salts of neodymium and ytterbium. Roughly the same cubic fields, together with small rhombic terms give satisfactory agreement for $\mathrm{Pr}+++$ and $\mathrm{Yb}+++$, and for $\mathrm{Nd}+++$ at all but very low temperatures. At these temperatures, as pointed out by Van Vleck ${ }^{3}$, small departures from cubic symmetry can only have the effect of increasing the susceptibility of $\mathrm{Nd}+++$, whereas the experimental results are considerably lower than those predicted by a field of cubic symmetry. We find that if the rhombic field is made sufficiently large to lower the mean susceptibility of $\mathrm{Nd}+++$ to the observed value, all resemblance of the energy diagram to the cubic field. pattern is lost.

Details will be published elsewhere.

$$
\begin{aligned}
& \text { Imperial College of Science } \\
& \text { and Technology, } \\
& \text { London, S.W.7. } \\
& \text { June 2. } \\
& { }^{1} \text { Spedding, Hankin and Nutting, J. Chem. Phys., 5, } 191 \text { (1937), } \\
& \text { and earlier papers. } \\
& { }^{2} \text { Krishnan and Mookherji, to appear shortly in Phil. Trans. } \\
& { }^{3} J . \text { Chem. Phys., 5, } 198(1937) .
\end{aligned}
$$

\section{Band Spectrum of Chromium Hydride, $\mathrm{CrH}$.}

ConTINUING our investigations of the spectra of the hydrides of the transition elements, we have now observed a band in the region $3600-3700 \mathrm{~A}$. when running a high-tension arc between chromium electrodes in a flame of hydrogen burning in air.

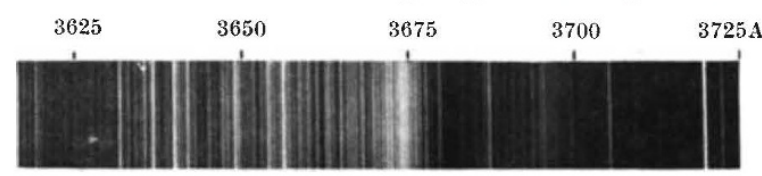

Fig. 1.

The method of production of this band is similar to that used by us to obtain the spectra of nickel hydride $^{1}, \mathrm{NiH}$, and manganese hydride ${ }^{2}, \mathrm{MnH}$, and we attribute the new band to $\mathrm{CrH}$. A spectrogram taken in the first order of a $20 \mathrm{ft}$. concave grating. is reproduced in Fig. 1. The band is degraded to shorter wave-lengths, and the rotational structure is only partially resolved under this dispersion.

The chromium oxide, CrO, bands in the orange region of the spectrum were not observed under the conditions most favourable for the production of the new band, and it seems very unlikely, for experimental reasons, that the band can be due to anything except the hydride. The chromium atom has a ${ }^{7} S$ level as its lowest state, and a high multiplicity, probably six, is therefore to be expected for the hydride; this would account for the complexity of the rotational structure of the band.

$$
\begin{array}{cl}
\text { Imperial College of Science, } & \text { A. G. Gaydor. } \\
\text { London, S.W.7. } & \text { R. W. B. PEARSE. }
\end{array}
$$

${ }^{1}$ Proc. Roy. Soc., A, 143, 312 (1935).

${ }^{2}$ NATURE, 139, 590 (1937).

\section{Nuclear Moments of Aluminium}

The hyperfine structure of the lines $3{ }^{2} P_{1 / 2}-4 S_{1 / 2}$ (3944 A.), $3{ }^{2} P_{3 / 2}-4 S_{1 / 2}$ (3961 A.), $3{ }^{2} P_{1 / 2}-3{ }^{2} D_{3 / 2}$ (3082 A.) and $3{ }^{2} P_{3 / 2}-3{ }^{2} D_{5 / 2}(3093 \mathrm{~A}$.) was investigated by means of the absorption in an atomic beam of aluminium. The structure of the lines $3{ }^{2} P_{3 / 2}-4 S_{1 / 2}$ and $3{ }^{2} P_{3 / 2}-3{ }^{2} D_{5 / 2}$ could not be resolved. The line $3{ }^{2} P_{1 / 2}-4 S_{1 / 2}$ was found to possess three components at $-0 \cdot 048,0 \cdot 000$ and $+0 \cdot 048 \mathrm{~cm}^{-1}$ of approximately equal intensity. The line $3^{2} P_{1 / 2}-$ $3{ }^{2} D_{3 / 2}$ possessed two components, their separation being $0.062 \mathrm{~cm}^{-1}$; the intensity ratio of these two components was measured to a high degree of accuracy; the mean of eighty measurements gave the value $1 \cdot 21$, the component of longer wave-length being the stronger.

From the observed structure of the line $3{ }^{2} P_{1 / 2}-$ $4 S_{1 / 2}$, it follows that the levels $3{ }^{2} P_{1 / 2}$ and $4 S_{1 / 2}$ are both split into two levels, of separation $0.048 \mathrm{~cm} .^{-1}$ (the greater splitting of the line $3{ }^{2} P_{1 / 2}-3{ }^{2} D_{3 / 2}$ is due to a small unresolvable inverted structure of the level $3{ }^{2} D_{3 / 2}$ which is probably caused by perturbation by the term $3 s 3 p^{2}{ }^{2} D_{3 / 2}$ ). The observed intensity ratio of the components of $3082 \mathrm{~A}$. gives a value $9 / 2$ for the nuclear spin, the theoretical value for a spin $9 / 2$ being $1 \cdot 22$. The magnetic moment, calculated from Goudsmit's formula, is $4 \cdot 1$ nuclear magnetons according to the splitting of the level $4 S_{1 / 2}$, and 3.6 nuclear magnetons according to the splitting of the level $3{ }^{2} P_{1 / 2}$. The agreement between these two values is the more satisfactory as the formula for $p$-terms gives, according to Goudsmit, rather too low values for light elements.

The lines $4 \quad S_{1 / 2}-5 \quad{ }^{2} P_{3 / 2} \quad(6696$ A.) and $4 S_{1 / 2}-5^{2} P_{1 / 2}(6699$ A.) were observed in emission; they were both doublets, only just resolvable on account of the Doppler width, of separation about $0.05 \mathrm{~cm}^{-1}$; the intensity ratio, when corrected for overlapping, was found to be about $1 \cdot 23$, the long wave component being the stronger. These observations are in agreement with the above conclusions.

This result is in disagreement with the contents of a preliminary note by Ritschl $^{1}$; from an observed doublet hyperfine structure in the lines 3057 and 3050 of the arc spectrum and 2669 of the spark spectrum, he suggested a nuclear spin of $1 / 2$; but he made no intensity measurements, and the observed doublet structures can be explained by incomplete resolution.
Clarendon Laboratory,
D. A. JACKsON.
Oxford.
H. KUHN.

May 14.

${ }^{1}$ Ritschl, R., NATURE, 131, 58 (1933). 